\title{
ANALYTIC MODEL OF THE SUBSYSTEM HEAD-NECK-SPINE MOVEMENT BIOMECHANICS
}

\section{Valentin AMORTILA, Elena MEREUTA, Monica NOVETSCHI, Madalina RUS, Silvia VEREȘIU}

\author{
"Dunarea de Jos" University, Galati, ROMANIA \\ valentin.amortila@ugal.ro
}

\begin{abstract}
Biomechanical modeling with the help of analytical mechanics together with the application of dedicated IT resources and mechanical simulations lead to a higher accuracy of the biomechanical model. From an analytical point of view, this article develops the equation of motion of the human head in a biomechanical system with three degrees of freedom using the Lagrange equations of the second case. It has been considered that this biomechanical subsystem consists of 3 anatomical elements: the head, the cervical region and the rest of the spine (considered as being a single element consisting of the thoracic region, lumbar region, sacral region and coccygeal region).

The forces which stress the head and spine during normal movements or when activities in the workplace are performed make biomechanical modeling different. Therefore, the movement of the subsystem was analyzed in the sagittal plane taking into consideration joints of cylindroid type, and the subsystem as a triple inverted pendulum.

The main purpose of this model was to obtain the differential equations of motion of the mechanical subsystem considered to be in the matrix-analytical form.
\end{abstract}

Keywords: biomechanical system, biomechanical model, Lagrange equations.

\section{INTRODUCTION}

Biomechanical analysis of the human body for identifying the equations of motion of anatomical elements or joints is and will remain a challenge for any researcher. The forces which stress the head and the spine during normal movements or when activities in the workplace are performed make biomechanical modeling different. The different interpretation of the origin and insertions of muscles (as the main generator of movement) for some biomechanical models belonging to the human body increase the complexity of explaining the phenomena. Modeling with the help of analytical mechanics together with the application of computer tools dedicated to mechanical simulations lead to a higher accuracy of the biomechanical model.

The development of a mathematical model of the skeletal-muscular system is based on a simplified representation of reality. The usefulness of the model lies in the ease of being used and implemented in computer applications in order to determine some biomechanical characteristics in a non-invasive way. At the same time, it must contain all the characteristic elements of the studied problem which can be modeled using the abstraction through which it is created a simplified image of the real system. The study of the joint mobility of the upper body requires the study of the movements of the head and spine. The achievement of a complete model of the head - spine subsystem implies an approach specific to each researcher.

The authors considered that this biomechanical subsystem consists of 3 general anatomical elements: the head, the cervical region and the rest of the spine considered as being a single element (thoracic region, lumbar region, sacral region and coccygeal region) (Fig. 1). 


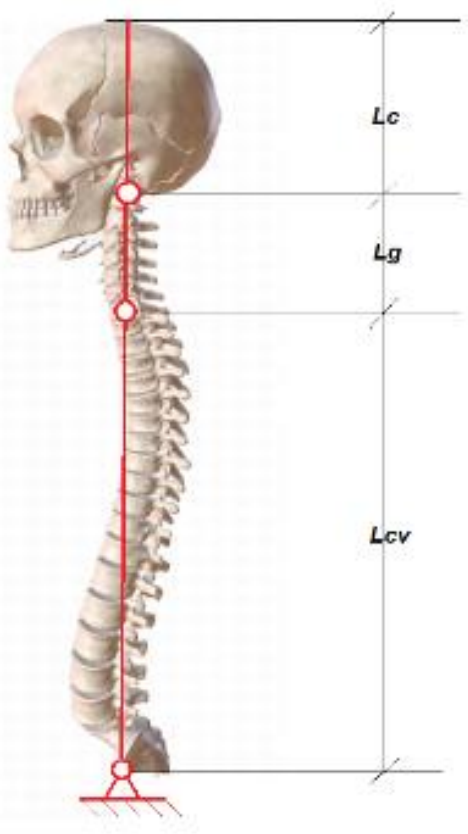

Fig. 1 Subsystem head, neck, spine

The anthropometric quantities of the three elements:

\begin{tabular}{|c|c|c|c|}
\hline \multirow[b]{2}{*}{ Segment } & \multicolumn{3}{|c|}{ Dimension } \\
\hline & $\begin{array}{l}\text { Length of } \\
\text { the element }\end{array}$ & $\begin{array}{c}\text { Distance } \\
\text { towards } \\
\text { the } \\
\text { proximal } \\
\text { joint of } \\
\text { the center } \\
\text { of gravity }\end{array}$ & $\begin{array}{l}\text { Total mass } \\
\text { percentage }\end{array}$ \\
\hline Spine & $\begin{array}{c}\mathrm{LcV}= \\
0.288 \cdot \mathrm{H}\end{array}$ & $\begin{array}{c}\mathrm{a}_{1}= \\
0.5 \cdot \mathrm{Lcv}\end{array}$ & $\begin{array}{c}\mathrm{m}_{1}= \\
35.5 \%\end{array}$ \\
\hline Neck & $\mathrm{Lg}=0.052 \cdot \mathrm{H}$ & $\begin{array}{c}\mathrm{a}_{2}= \\
0.5 \cdot \mathrm{Lg}\end{array}$ & $\mathrm{m}_{2}=1.5 \%$ \\
\hline Head & $\mathrm{Lc}=0.130 \cdot \mathrm{H}$ & $\begin{array}{c}\mathrm{a}_{3}= \\
0.5 \cdot \mathrm{Lc}\end{array}$ & $\mathrm{m}_{3}=6.6 \%$ \\
\hline
\end{tabular}

Lcv - the length of the spine,

$\mathrm{Lg}$-the length of the neck,

$\mathrm{Lc}$ - the length of the head,

$a_{1}$ - the proximal distance of the center of gravity of the spine (element 1),

$\mathrm{a}_{2}$ - the distance towards the proximal joint of the center of gravity of the neck (element 2),

$\mathrm{a}_{3}$ - the distance towards the proximal joint of the center of gravity of the head (element 3 ),

$\mathrm{m}_{1}$ - trunk mass (mass of element 1 ),

$\mathrm{m}_{2}-$ neck mass (mass of element 2 ),

$\mathrm{m}_{3}-$ head mass (mass of element 3 ).

The masses and position of the center of gravity have been estimated for the three elements - head, neck and spine - starting from the total mass and the dimensions of the segments (through anthropometric measurements) [1], [2], [3].

\section{THE METHOD USED IN BIOMECHANICAL MODELING OF THE HEAD - SPINE SUBSYSTEM}

In order to analyze this subsystem from the point of view of a mechanical model, the authors considered the subsystem as a triple inverted physical pendulum consisting of 2 bars articulated with (spine and neck) and a sphere (head), (Fig. 2).

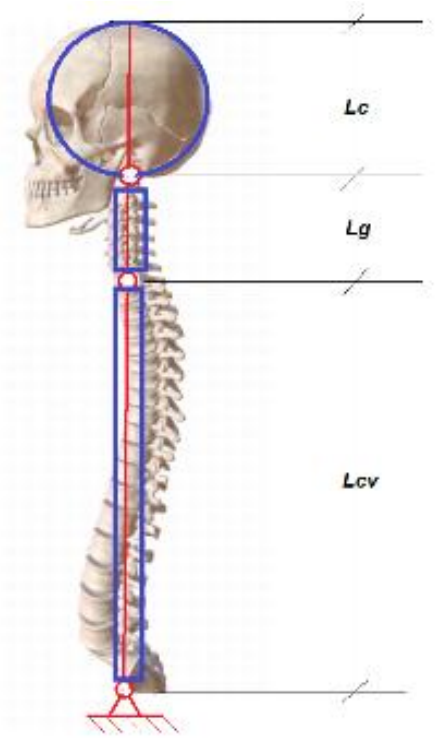

Fig. 2 The transformation of the subsystem into a physical model - triple inverted pendulum

For the presented model, it was considered that the three elements are articulated with cylindrical joints (hinge) that allow rotational movement only in the sagittal plane. There have not been taken into consideration any possible slips in the anatomical joints. Thus, the proposed physical model has 3 degrees of freedom on which three disturbing forces F1, F2, F3 act, that perform in the centers of gravity of the elements, forces which disturb the orthostatic position (Fig. 3a and Fig. 3b).

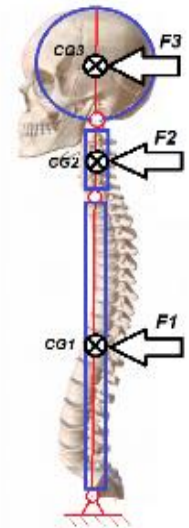

(a)

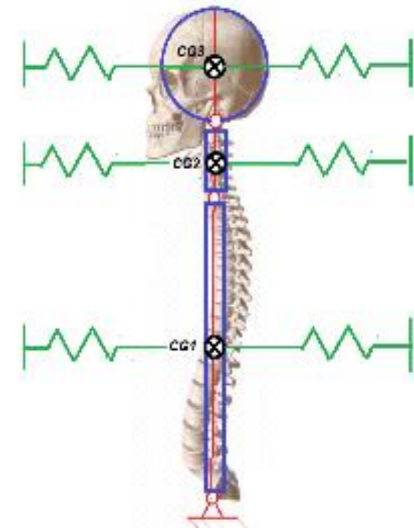

(b)
Fig. 3 Forces which disturb the orthostatic position 
The coordinates of the center of gravity belonging to element 1, CG1 (Fig. 4) [4]:

$$
\begin{aligned}
& x_{C G 1}=a_{1} \sin \theta_{1} \\
& y_{C G 1}=a_{1} \cos \theta_{1}
\end{aligned}
$$

The coordinates of the center of gravity belonging to element $2, \mathrm{CG}_{2}$ :

$$
\begin{aligned}
& x_{C G 2}=L_{c v} \sin \theta_{1}+a_{2} \sin \theta_{2} \\
& y_{C G 2}=L_{c v} \cos \theta_{1}+a_{2} \cos \theta_{2}
\end{aligned}
$$

The coordinates of the center of gravity belonging to element $3, \mathrm{CG}_{3}$ :

$$
\begin{gathered}
x_{C G 3}=L_{c v} \sin \theta_{1}+L_{g} \sin \theta_{2}+a_{3} \sin \theta_{3} \\
y_{C G 3}=L_{c v} \cos \theta_{1}+L_{g} \cos \theta_{2}+a_{3} \cos \theta_{3}
\end{gathered}
$$

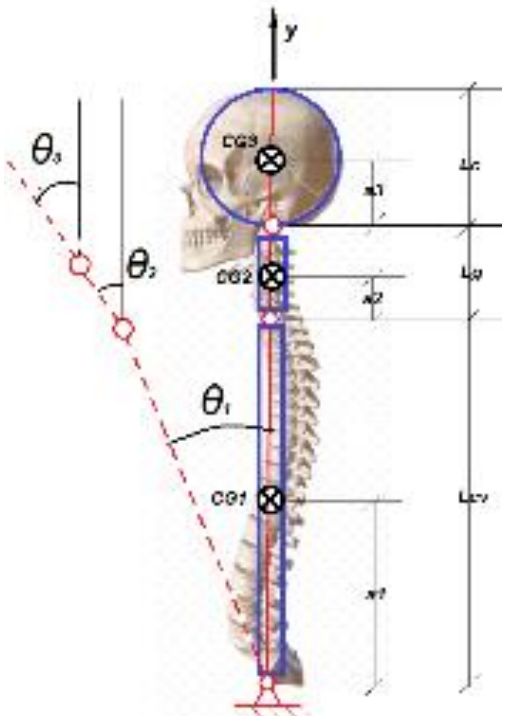

Figure 4 The coordinates of the center of gravity

Velocity determination of the center of gravity of element $2, \mathrm{CG}_{2}$, is obtained by deriving the displacement of the center of gravity:

Velocity determination of the center of gravity of element 2, CG2, is obtained by deriving the displacement of the center of gravity:

$$
\begin{aligned}
& \dot{x}_{C G 2}=L_{c v} \dot{\theta}_{1} \cos \theta_{1}+a_{2} \dot{\theta}_{2} \cos \theta_{2} \\
& \dot{y}_{C G 2}=L_{c v} \dot{\theta}_{1}\left(-\sin \theta_{1}\right)+a_{2} \dot{\theta}_{2}\left(-\sin \theta_{2}\right) \\
& v_{C G 2}^{2}=\dot{x}_{C G 2}{ }^{2}+\dot{y}_{C G 2}{ }^{2}=\left(L_{c v} \dot{\theta}_{1} \cos \theta_{1}\right)^{2}+ \\
& +2 \cdot\left(L_{c v} \dot{\theta}_{1} a_{2} \dot{\theta}_{2} \cos \theta_{1} \cos \theta_{2}\right)+ \\
& +\left(a_{2} \dot{\theta}_{2} \cos \theta_{2}\right)^{2}+\left(L_{c v} \dot{\theta}_{1}\left(-\sin \theta_{1}\right)\right)^{2}+ \\
& +2 \cdot L_{c v} \dot{\theta}_{1}\left(-\sin \theta_{1}\right) a_{2} \dot{\theta}_{2}\left(-\sin \theta_{2}\right)+ \\
& \left(a_{2} \dot{\theta}_{2}\left(-\sin \theta_{2}\right)\right)^{2}=2 \cdot L_{c v}^{2} \dot{\theta}_{1}^{2}+ \\
& +2 L_{c v} a_{2} \dot{\theta}_{1} \dot{\theta}_{2} \cos \left(\theta_{2}-\theta_{1}\right)+a_{2} \dot{\theta}_{2}^{2}
\end{aligned}
$$

Velocity determination of the center of gravity of element 3, CG3:

$$
\dot{x}_{C G 3}=L_{c v} \dot{\theta}_{1} \cos \theta_{1}+L_{g} \dot{\theta}_{2} \cos \theta_{2}+a_{3} \dot{\theta}_{3} \cos \theta_{3}
$$

$$
\begin{gathered}
\dot{y}_{C G 3}=L_{c v} \dot{\theta}_{1}\left(-\sin \theta_{1}\right)+L_{g} \dot{\theta}_{2}\left(-\sin \theta_{2}\right)+ \\
+a_{3} \dot{\theta}_{3}\left(-\sin \theta_{3}\right) \\
v_{C G 3}^{2}=\dot{x}_{C G 3}{ }^{2}+\dot{y}_{C G 3}{ }^{2}=\left(L_{c v} \dot{\theta}_{1} \cos \theta_{1}\right)^{2}+ \\
+\left(L_{g} \dot{\theta}_{2} \cos \theta_{2}\right)^{2}+\left(a_{3} \dot{\theta}_{3} \cos \theta_{3}\right)^{2}+ \\
+2\left(L_{c v} \dot{\theta}_{1} L_{g} \dot{\theta}_{2} \cos \theta_{1} \cos \theta_{2}\right)+ \\
+2\left(L_{c v} \dot{\theta}_{1} a_{3} \dot{\theta}_{3} \cos \theta_{1} \cos \theta_{3}\right)+ \\
+2\left(L_{g} \dot{\theta}_{2} a_{3} \dot{\theta}_{3} \cos \theta_{2} \cos \theta_{3}\right)+ \\
+\left(L_{c v} \dot{\theta}_{1}\left(-\sin \theta_{1}\right)\right)^{2}+\left(L_{g} \dot{\theta}_{2}\left(-\sin \theta_{2}\right)\right)^{2}+ \\
+\left(a_{3} \dot{\theta}_{3}\left(-\sin \theta_{3}\right)\right)^{2}+ \\
+2\left(L_{c v} \dot{\theta}_{1}\left(-\sin \theta_{1}\right) L_{g} \dot{\theta}_{2}\left(-\sin \theta_{2}\right)\right)+ \\
+2\left(L_{c v} \dot{\theta}_{1}\left(-\sin \theta_{1}\right) a_{3} \dot{\theta}_{3}\left(-\sin \theta_{3}\right)\right)+ \\
+2\left(L_{g} \dot{\theta}_{2}\left(-\sin \theta_{2}\right) a_{3} \dot{\theta}_{3}\left(-\sin \theta_{3}\right)\right)= \\
=L_{c v}^{2} \dot{\theta}_{1}^{2}+L_{g}^{2} \dot{\theta}_{2}^{2}+a_{3}^{2} \dot{\theta}_{3}^{2}+ \\
+2 L_{c v} L_{g} \dot{\theta}_{1} \dot{\theta}_{2} \cos \left(\theta_{2}-\theta_{1}\right)+ \\
+L_{c v} a_{3} \dot{\theta}_{1} \dot{\theta}_{3} \cos \left(\theta_{3}-\theta_{1}\right)+L_{g} a_{3} \dot{\theta}_{2} \dot{\theta}_{3} \cos \left(\theta_{3}-\theta_{2}\right)
\end{gathered}
$$

The equation of motion for the proposed model

According to the Euler-Lagrange equations of the second case:

$$
\frac{d}{d t}\left(\frac{\partial E_{C}}{\partial \dot{q}_{i}}\right)-\left(\frac{\partial E_{C}}{\partial q_{i}}\right)=F_{i P}
$$

where:

$q_{i}-$ generalized coordinates of the system which determines the system of differential equations for the proposed model $\left(q_{1}=\theta_{1}, q_{2}=\theta_{2}, q_{3}=\theta_{3}\right)$,

$E_{C}$ - kinetic energy of the mechanical system which characterizes the state of motion of solid bodies system considered rigid, with connections,

$F_{i P}$ - generalized disturbing forces.

Disturbing forces are precisely the forces that generate the movement of anatomical elements. These forces are developed by the muscles responsible for each movement. The muscle behaves like a spring, which generates the movement when it contracts (agonist muscles), a movement which is moderated by the action of other muscles that oppose the movement (antagonistic muscles).

The determination of kinetic energy of the mechanical system composed of the 3 solid bodies

Kinetic energy of the analyzed subsystem consists of the rotational kinetic energy and the translational kinetic energy of each element:

$$
\begin{gathered}
E_{C}=E c_{\text {rot }}+E c_{\text {tran }}= \\
=E_{1}+E_{2}+E_{3}+E_{C G 2}+E_{C G 3}
\end{gathered}
$$

where:

$E_{1}=\frac{1}{2} J_{O 1} \omega_{1}=\frac{1}{2} J_{O 1} \dot{\theta}_{1}^{2}$ (the rotational kinetic energy of body 1 ) 
$E_{2}=\frac{1}{2} J_{O 2} \omega_{2}=\frac{1}{2} J_{O 2} \dot{\theta}_{2}^{2}$ (the rotational kinetic energy of body 2),

$E_{3}=\frac{1}{2} J_{O 3} \omega_{3}=\frac{1}{2} J_{O 3} \dot{\theta}_{3}^{2}$ (the rotational kinetic energy of body 3$)$,

$E_{c g 2}=\frac{1}{2} m_{2} v_{C M 2}^{2}$ (the translational kinetic energy of body 2),

$E_{c g 3}=\frac{1}{2} m_{3} v_{C M 3}^{2}$ (the rotational kinetic energy of body 3 ).

\section{The determination of the moments of inertia of the}

\section{3 solid bodies:}

The three articulated anatomical elements were considered homogeneous bodies having the mechanical interpretation as follows:

- the spine was considered a homogeneous bar whose moment of inertia $J_{O I}$ is:

$$
J_{O 1}=\frac{m_{1} L_{c v}^{2}}{3}
$$

- the cervical region (neck) was considered a homogeneous bar whose moment of inertia $J_{O 2}$ is:

$$
J_{O 2}=\frac{m_{2} L_{g}^{2}}{12}
$$

- the head was considered a sphere whose moment of inertia $J_{O 3}$ is:

$$
J_{O 3}=\frac{2}{5} m_{3} R_{C}^{2}=\frac{1}{10} m_{3} L_{C}^{2}
$$

where:

$R_{C}=L_{C} / 2=0.65 \cdot H-$ the radius of the sphere that physically shapes the head

The kinetic energy of the subsystem becomes:

$$
\begin{array}{r}
E_{C}=\frac{1}{2} \frac{m_{1} L_{c v}^{2}}{3} \dot{\theta}_{1}^{2}+\frac{1}{2} \frac{m_{2} L_{g}^{2}}{12} \dot{\theta}_{2}^{2}+\frac{1}{2} \frac{1}{10} m_{3} L_{C}^{2} \dot{\theta}_{3}^{2}+ \\
+\frac{1}{2} m_{2} \cdot\left[2 L_{c v}^{2} \dot{\theta}_{1}^{2}+2 L_{c v} a_{2} \dot{\theta}_{1} \dot{\theta}_{2} \cos \left(\theta_{2}-\theta_{1}\right)+\right. \\
\left.a_{2} \dot{\theta}_{2}^{2}\right]+\frac{1}{2} m_{3} \cdot\left[L_{c v}^{2} \dot{\theta}_{1}^{2}+L_{g}^{2} \dot{\theta}_{2}^{2}+a_{3}^{2} \dot{\theta}_{3}^{2}+\right. \\
2 L_{c v} L_{g} \dot{\theta}_{1} \dot{\theta}_{2} \cos \left(\theta_{2}-\theta_{1}\right)+L_{c v} a_{3} \dot{\theta}_{1} \dot{\theta}_{3} \cos \left(\theta_{3}-\right. \\
\left.\left.\theta_{1}\right)+L_{g} a_{3} \dot{\theta}_{2} \dot{\theta}_{3} \cos \left(\theta_{3}-\theta_{2}\right)\right]=\frac{1}{2}\left(\frac{m_{1} L_{c v}^{2}}{3}+\right. \\
\left.+2 m_{2} L_{c v}^{2}+m_{3} L_{c v}^{2}\right) \dot{\theta}_{1}^{2}+\frac{1}{2}\left(\frac{m_{2} \cdot L_{g}^{2}}{12}+m_{2} a_{2}+\right. \\
\left.\quad+m_{3} L_{g}^{2}\right) \dot{\theta}_{2}^{2}+\frac{1}{2}\left(\frac{1}{5} m_{3} L_{C}^{2}+m_{3} a_{3}^{2}\right) \dot{\theta}_{3}^{2}+ \\
+\frac{1}{2} \cdot\left[2 m_{2} L_{c v} a_{2} \cos \left(\theta_{2}-\theta_{1}\right)+2 m_{3} L_{c v} L_{g} \cos \left(\theta_{2}-\right.\right. \\
\left.\left.-\theta_{1}\right)\right] \dot{\theta}_{1} \dot{\theta}_{2}+\frac{1}{2} \cdot\left[m_{3} L_{c v} a_{3} \cos \left(\theta_{3}-\theta_{1}\right)\right] \dot{\theta}_{1} \dot{\theta}_{3}+ \\
+\frac{1}{2} \cdot\left[m_{3} L_{g} a_{3} \cos \left(\theta_{3}-\theta_{2}\right)\right] \dot{\theta}_{2} \dot{\theta}_{3}
\end{array}
$$

meaning,

$$
E_{C}=\frac{1}{2}\left(m_{11} \dot{\theta}_{1}^{2}+m_{22} \dot{\theta}_{2}^{2}+m_{33} \dot{\theta}_{3}^{2}+2 m_{12} \dot{\theta}_{1} \dot{\theta}_{2}+\right.
$$$$
\left.+2 m_{13} \dot{\theta}_{1} \dot{\theta}_{3}+2 m_{23} \dot{\theta}_{2} \dot{\theta}_{3}\right)
$$

Comparing the general form of the kinetic energy with the kinetic energy of the analyzed subsystem, [5] there can be determined the terms of the inertia matrix $[M]$ :

$$
\begin{aligned}
m_{11}=\frac{m_{1} L_{c v}^{2}}{3} & +2 m_{2} L_{c v}^{2}+m_{3} L_{c v}^{2} \\
m_{12}=m_{2} L_{c v} a_{2} \cos \left(\theta_{2}-\theta_{1}\right)+ & + \\
& +m_{3} L_{c v} L_{g} \cos \left(\theta_{2}-\theta_{1}\right)
\end{aligned}
$$

$$
\begin{gathered}
m_{13}=\frac{1}{2} m_{3} L_{c v} a_{3} \cos \left(\theta_{3}-\theta_{1}\right) \\
m_{21}=m_{2} L_{c v} a_{2} \cos \left(\theta_{2}-\theta_{1}\right)+ \\
+m_{3} L_{c v} L_{g} \cos \left(\theta_{2}-\theta_{1}\right) \\
m_{22}=\frac{m_{2} L_{g}^{2}}{12}+m_{2} a_{2}+m_{3} L_{g}^{2} \\
m_{23}=\frac{1}{2} m_{3} L_{g} a_{3} \cos \left(\theta_{3}-\theta_{2}\right) \\
m_{31}=\frac{1}{2} m_{3} L_{c v} a_{3} \cos \left(\theta_{3}-\theta_{1}\right) \\
m_{32}=\frac{1}{2} m_{3} L_{g} a_{3} \cos \left(\theta_{3}-\theta_{2}\right) \\
m_{33}=\frac{1}{5} m_{3} L_{C}^{2}+m_{3} a_{3}^{2}
\end{gathered}
$$

Determination of the potential energy of mechanical system composed of 3 solid bodies and 3 springs

The potential energy of the analyzed subsystem consists of the potential energy of each element and the potential energies of the limitative springs [6], [7]:

$$
E_{P}=E_{p 1}+E_{p 2}+E_{p 3}+2 E_{p a 1}+2 E_{p a 2}+2 E_{p a 3}
$$

where:

$E_{p 1}=m_{1} g \Delta h_{1}=($ potential energy of body 1$)$, $E_{p 2}=m_{2} g \Delta h_{2}$ (potential energy of body 2$)$, $E_{p 3}=m_{3} g \Delta h_{3}$ (potential energy of body 3$)$ where:

$$
\begin{gathered}
\Delta h_{1}=a_{1}\left(1-\cos \theta_{1}\right) \\
\Delta h_{2}=L_{c v}\left(1-\cos \theta_{1}\right)+a_{2}\left(1-\cos \theta_{2}\right) \\
\Delta h_{3}=L_{c v}\left(1-\cos \theta_{1}\right)+L_{g}\left(1-\cos \theta_{2}\right)+ \\
+a_{3}\left(1-\cos \theta_{3}\right)
\end{gathered}
$$

meaning

$$
\begin{gathered}
E_{p 1}=m_{1} g \Delta h_{1}=m_{1} g a_{1}\left(1-\cos \theta_{1}\right) \\
E_{p 2}=m_{2} g \Delta h_{2}=
\end{gathered}
$$$$
=m_{2} g\left[L_{c v}\left(1-\cos \theta_{1}\right)+a_{2}\left(1-\cos \theta_{2}\right)\right]
$$$$
E_{p 3}=m_{3} g \Delta h_{3}=m_{3} g\left[L_{c v}\left(1-\cos \theta_{1}\right)+\right.
$$$$
\left.+L_{g}\left(1-\cos \theta_{2}\right)+a_{3}\left(1-\cos \theta_{3}\right)\right]
$$

For small values of angle $\theta$, the substitution $\sin \theta \approx \theta$ is accepted, meaning $\sin \frac{\theta}{2} \approx \frac{\theta}{2}$ resulting: $\cos \theta=1-2 \sin ^{2} \frac{\theta}{2} \rightarrow 1-\cos \theta=2 \sin ^{2} \frac{\theta}{2}=$ $=2 \frac{\theta^{2}}{4} \rightarrow 1-\cos \theta \approx \frac{\theta^{2}}{2}$

$$
\begin{gathered}
E_{p 1}=\frac{1}{2} m_{1} g a_{1} \theta_{1}^{2} \\
E_{p 2}=\frac{1}{2} m_{2} g\left(L_{c v} \theta_{1}^{2}+a_{2} \theta_{2}^{2}\right) \\
E_{p 3}=\frac{1}{2} m_{3} g\left(L_{c v} \theta_{1}^{2}+L_{g} \theta_{2}^{2}+a_{3} \theta_{3}^{2}\right)
\end{gathered}
$$

$E_{p a 1}=\frac{1}{2} k_{1}\left(x_{i}^{2}-x_{f 1}^{2}\right)$ (potential energy of spring 1$)$, $E_{p a 2}=\frac{1}{2} k_{2}\left(x_{i}^{2}-x_{f 2}^{2}\right)$ (potential energy of spring 2), $E_{p a 3}=\frac{1}{2} k_{3}\left(x_{i}^{2}-x_{f 3}^{2}\right)$ (potential energy of spring 3 ), where:

$k_{i}$ - the coefficient of elasticity of the spring ,i” (muscle), $x_{i}$ - the initial length of the spring, $x_{f 1}-$ the final length of spring 1 , 
$x_{f 2}-$ the final length of spring 2 ,

$x_{f 3}-$ the final length of spring 3 ,

The initial lengths of the springs are:

$$
\begin{aligned}
& x_{i}=x_{f 1}+\delta_{1} \\
& x_{i}=x_{f 2}+\delta_{2} \\
& x_{i}=x_{f 3}+\delta_{3}
\end{aligned}
$$

where:

$\delta_{1}=a_{1} \sin \theta_{1}-$ the displacement of spring 1 ,

$\delta_{1}=a_{2} \sin \theta_{2}+L_{c v} \sin \theta_{1}-$ the displacement of spring 2 ,

$\delta_{3}=a_{3} \sin \theta_{3}+L_{g} \sin \theta_{2}+L_{c v} \sin \theta_{1} \quad-\quad$ the displacement of spring 3 ,

For small values of angle $\theta$, the substitution $\sin \theta \approx \theta$ is accepted, meaning

$$
\begin{gathered}
\delta_{1}=a_{1} \theta_{1} \\
\delta_{1}=a_{2} \theta_{2}+L_{c v} \theta_{1} \\
\delta_{3}=a_{3} \theta_{3}+L_{g} \theta_{2}+L_{c v} \theta_{1}
\end{gathered}
$$

Therefore, potential energies of the springs become [8]:

$$
\begin{aligned}
& E_{p a 1}=\frac{1}{2} k_{1}\left(x_{f 1}^{2}+2 x_{f 1} \delta_{1}+\delta_{1}^{2}-x_{f 1}^{2}\right) \\
& E_{p a 1}=\frac{1}{2} k_{1}\left(2 x_{f 1} a_{1} \theta_{1}+a_{1}^{2} \theta_{1}^{2}\right) \\
& E_{p a 2}=\frac{1}{2} k_{2}\left(x_{f 2}^{2}+2 x_{f 2} \delta_{2}+\delta_{2}^{2}-x_{f 2}^{2}\right) \\
& E_{p a 2}=\frac{1}{2} k_{2}\left(2 x_{f 2} a_{2} \theta_{2}+2 L_{c v} \theta_{1}+a_{2}^{2} \theta_{2}^{2}+\right. \\
& \left.+2 a_{2} L_{c v} \theta_{1} \theta_{2}+L_{c v}^{2} \theta_{1}^{2}\right) \\
& E_{p a 3}=\frac{1}{2} \cdot k_{3} \cdot\left(x_{f 3}^{2}+2 x_{f 3} \delta_{3}+\delta_{3}^{2}-x_{f 3}^{2}\right) \\
& E_{p a 3}=\frac{1}{2} k_{3}\left(2 x_{f 3} a_{3} \theta_{3}+2 x_{f 3} L_{g} \theta_{2}+2 x_{f 3} L_{c v} \theta_{1}+\right. \\
& +a_{3}^{2} \theta_{3}^{2}+L_{g}^{2} \theta_{2}^{2}+L_{c v}^{2} \theta_{1}^{2}+ \\
& \left.+2 a_{3} L_{g} \theta_{2} \theta_{3}+2 a_{3} L_{c v} \theta_{1} \theta_{3}+2 L_{g} L_{c v} \theta_{1} \theta_{2}+L_{c v}^{2} \theta_{1}^{2}\right)
\end{aligned}
$$

Substituting in relation (11), the potential energy of the system can be written:

$$
\begin{aligned}
& E_{P}=\frac{1}{2} m_{1} g a_{1} \theta_{1}^{2}+\frac{1}{2} m_{2} g\left(L_{c v} \theta_{1}^{2}+a_{2} \theta_{2}^{2}\right)+ \\
& \frac{1}{2} m_{3} g\left(L_{c v} \theta_{1}^{2}+L_{g} \theta_{2}^{2}+a_{3} \theta_{3}^{2}\right)+2 \frac{1}{2} k_{1}\left(2 x_{f 1} a_{1} \theta_{1}+\right. \\
& \left.a_{1}^{2} \theta_{1}^{2}\right)+2 \frac{1}{2} k_{2}\left(2 x_{f 2} a_{2} \theta_{2}+2 L_{c v} \theta_{1}+a_{2}^{2} \theta_{2}^{2}+\right. \\
& \left.2 a_{2} L_{c v} \theta_{1} \theta_{2}+L_{c v}^{2} \theta_{1}^{2}\right)+2 \frac{1}{2} k_{3}\left(2 x_{f 3} a_{3} \theta_{3}+\right. \\
& 2 x_{f 3} L_{g} \theta_{2}+2 x_{f 3} L_{c v} \theta_{1}+a_{3}^{2} \theta_{3}^{2}+L_{g}^{2} \theta_{2}^{2}+L_{c v}^{2} \theta_{1}^{2}+ \\
& \left.2 a_{3} L_{g} \theta_{2} \theta_{3}+2 a_{3} L_{c v} \theta_{1} \theta_{3}+2 L_{g} L_{c v} \theta_{1} \theta_{2}+L_{c v}^{2} \theta_{1}^{2}\right) \\
& E_{P}=\frac{1}{2}\left[m_{1} g a_{1}+m_{2} g L_{c v}+m_{3} g L_{c v}+2 k_{1} a_{1}^{2}+\right. \\
& \left.+2 k_{2} L_{c v}^{2}+4 k_{3} L_{c v}^{2}\right] \theta_{1}^{2}++\frac{1}{2}\left[m_{2} g a_{2}+m_{3} g L_{g}+\right. \\
& \left.+2 k_{2} a_{2}^{2}+2 k_{3} L_{g}^{2}\right] \theta_{2}^{2}+\frac{1}{2}\left[m_{3} g a_{3}+2 k_{3} a_{3}^{2}\right] \theta_{3}^{2}+ \\
& +\frac{1}{2}\left[4 k_{2} a_{2} L_{c v}+4 k_{3} L_{g} L_{c v}\right] \theta_{1} \theta_{2}+ \\
& +\frac{1}{2}\left[4 k_{3} a_{3} L_{g}\right] \theta_{2} \theta_{3}+\frac{1}{2}\left[4 k_{3} a_{3} L_{c v}\right] \theta_{1} \theta_{3}+ \\
& +\frac{1}{2}\left[4 k_{1} x_{f 1} a_{1}+4 k_{2} L_{c v}+4 k_{3} x_{f 3} L_{c v}\right] \theta_{1}+ \\
& +\frac{1}{2}\left[4 k_{2} x_{f 2} a_{2}+4 k_{3} x_{f 3} L_{g}\right] \theta_{2}+\frac{1}{2}\left[4 k_{3} x_{f 3} a_{3}\right] \theta_{3}
\end{aligned}
$$

In turn, deriving the relation of the potential energy of the system according to the three generalized coordinates, $\theta_{1}, \theta_{2}, \theta_{3}$, and analyzing for the initial position of the system when the generalized coordinates are equal to zero, it is obtained $x_{f i}=0$ and the potential energy becomes:

$E_{P}=\frac{1}{2}\left[m_{1} g a_{1}+m_{2} g L_{c v}+m_{3} g L_{c v}+2 k_{1} a_{1}^{2}+\right.$

$\left.2 k_{2} L_{c v}^{2}+4 k_{3} L_{c v}^{2}\right] \theta_{1}^{2}+\frac{1}{2}\left[m_{2} g a_{2}+m_{3} g L_{g}+\right.$

$\left.2 k_{2} a_{2}^{2}+2 k_{3} L_{g}^{2}\right] \theta_{2}^{2}+\frac{1}{2}\left[m_{3} g a_{3}+2 k_{3} a_{3}^{2}\right] \theta_{3}^{2}+$

$+\frac{1}{2}\left[4 k_{2} a_{2} L_{c v}+4 k_{3} L_{g} L_{c v}\right] \theta_{1} \theta_{2}+$

$+\frac{1}{2}\left[4 k_{3} a_{3} L_{g}\right] \theta_{2} \theta_{3}+\frac{1}{2}\left[4 k_{3} a_{3} L_{c v}\right] \theta_{1} \theta_{3}$

Identifying the terms in the general form of the potential energy, there are determined the terms of the rigidity matrix [5]:

$$
\begin{gathered}
k_{11}=m_{1} g a_{1}+m_{2} g L_{c v}+m_{3} g L_{c v}+ \\
+2 k_{1} a_{1}^{2}+2 k_{2} L_{c v}^{2}+4 k_{3} L_{c v}^{2} \\
k_{12}=2 k_{2} a_{2} L_{c v}+4 k_{3} L_{g} L_{c v} \\
k_{13}=2 k_{3} a_{3} L_{c v} \\
k_{21}=2 k_{2} a_{2} L_{c v}+4 k_{3} L_{g} L_{c v} \\
k_{22}=m_{2} g a_{2}+m_{3} g L_{g}+2 k_{2} a_{2}^{2}+ \\
+2 k_{3} L_{g}^{2} \\
k_{23}=2 k_{3} a_{3} L_{g} \\
k_{31}=2 k_{3} a_{3} L_{c v} \\
k_{32}=2 k_{3} a_{3} L_{g} \\
k_{33}=m_{3} g a_{3}+2 k_{3} a_{3}^{2}
\end{gathered}
$$

The ecuation of movement for the analyzed model can be written in matrix form [9]:

$$
[M]\left\{\begin{array}{l}
\ddot{\theta}_{1} \\
\ddot{\theta}_{2} \\
\ddot{\theta}_{3}
\end{array}\right\}+[K]\left\{\begin{array}{l}
\theta_{1} \\
\theta_{2} \\
\theta_{3}
\end{array}\right\}=\left[F_{i P}\right]
$$

and analytical form:

$$
\left\{\begin{array}{l}
\left(m_{11}+m_{12}+m_{13}\right) \ddot{\theta}_{1}+\left(k_{11}+k_{12}+k_{13}\right) \theta_{1}=F_{1} \\
\left(m_{21}+m_{22}+m_{23}\right) \ddot{\theta}_{2}+\left(k_{21}+k_{22}+k_{23}\right) \theta_{2}=F_{2} \\
\left(m_{31}+m_{32}+m_{33}\right) \ddot{\theta}_{3}+\left(k_{31}+k_{32}+k_{33}\right) \theta_{3}=F_{3}
\end{array}\right.
$$

Substituting the terms of the inertia matrix and those of the stiffness matrix, it is obtained the system of equations formed by the relations (13), (14), (15) which represent exactly the equations of the analyzed subsystem.

$$
\begin{gathered}
{\left[\frac{m_{1} L_{c v}^{2}}{3}+2 m_{2} L_{c v}^{2}+m_{3} L_{c v}^{2}+\right.} \\
+m_{2} L_{c v} a_{2} \cos \left(\theta_{2}-\theta_{1}\right)+m_{3} L_{c v} L_{g} \cos \left(\theta_{2}-\theta_{1}\right)+ \\
\left.+\frac{1}{2} m_{3} L_{c v} a_{3} \cos \left(\theta_{3}-\theta_{1}\right)\right] \ddot{\theta}_{1}+ \\
+\left(m_{1} g a_{1}+m_{2} g L_{c v}+m_{3} g L_{c v}+2 k_{1} a_{1}^{2}+\right. \\
+2 k_{2} L_{c v}^{2}+4 k_{3} L_{c v}^{2}+2 k_{2} a_{2} L_{c v}+4 k_{3} L_{g} L_{c v}+ \\
\left.+2 k_{3} a_{3} L_{c v}\right) \theta_{1}=F_{1}(13) \\
{\left[m_{2} L_{c v} a_{2} \cos \left(\theta_{2}-\theta_{1}\right)+m_{3} L_{c v} L_{g} \cos \left(\theta_{2}-\right.\right.} \\
\left.\theta_{1}\right)+\frac{m_{2} L_{g}^{2}}{12}+m_{2} a_{2}+m_{3} L_{g}^{2}+ \\
\left.+\frac{1}{2} m_{3} L_{g} a_{3} \cos \left(\theta_{3}-\theta_{2}\right)\right] \ddot{\theta}_{2}+
\end{gathered}
$$




$$
\begin{gathered}
+\left(2 k_{2} a_{2} L_{c v}+4 k_{3} L_{g} L_{c v}+m_{2} g a_{2}+m_{3} g L_{g}+\right. \\
\left.+2 k_{2} a_{2}^{2}+2 k_{3} L_{g}^{2}+2 k_{3} a_{3} L_{g}\right) \theta_{2}=F_{2} \\
{\left[\frac{1}{2} m_{3} L_{c v} a_{3} \cos \left(\theta_{3}-\theta_{1}\right)+\right.} \\
\left.+\frac{1}{2} m_{3} L_{g} a_{3} \cos \left(\theta_{3}-\theta_{2}\right)+\frac{1}{5} m_{3} L_{C}^{2}+m_{3} a_{3}^{2}\right] \ddot{\theta}_{3}+ \\
+\left(2 k_{3} a_{3} L_{c v}+2 k_{3} a_{3} L_{g}+m_{3} g a_{3}+2 k_{3} a_{3}^{2}\right) \theta_{3}=F_{3}
\end{gathered}
$$

\section{CONCLUSIONS}

The advantages of mathematical modeling stand out, especially in the case of complex biomechanical systems when it is necessary to determine a series of parameters using non-invasive methods. Thus, the investigation of a particular subsystem is possible if real experiments cannot be performed. At the same time, the use of the model allows iteration on various anthropometry whenever needed in order to perform an analysis as accurate as possible. Also, by modifying the parameters of the biomechanical system, the extreme values of the variables can be established, values which would correspond to some limit anatomical positions. It can be concluded that the study based on models is much more efficient both qualitatively and economically (low costs, reduced time, etc.)

The analytical model head-neck-spine can be used when the angular variations of the studied anatomical elements $\left(\theta_{1}, \theta_{2}, \theta_{3}\right)$ can be determined. This thing is possible due to a series of computer applications which use markers (Kinovea) or with the help of some sensors that can determine angular displacements.

The developed model can determine the values of the forces responsible for the movement of the head, neck or trunk, but also the restorative forces responsible for the return of the body to the orthostatic position.

For the future, we intend to make a parameterized analysis of this analytical model in order to establish the values of disturbing and/or restorative forces according to various anthropometries.

\section{ACKNOWLEDGEMENTS}

This work is supported by the project ANTREPRENORDOC, in the framework of Human Resources Development Operational Programme 2014-2020, financed from the European Social Fund under the contract number 36355/23.05.2019 HRD OP /380/6/13 - SMIS Code: 123847.

\section{REFERENCES}

[1] Budescu E., Dănilă C., Indrumar de lucrări aplicative. Biomecanică, 2013, https://mec.tuiasi.ro/diverse/Indrumar_lucrari_aplicat ive Biomecanica.pdf.

[2] Budescu, E., Biomecanica Generala, Iasi, 2013.

[3] Budescu, E., Iacob, I., Bazele biomecanicii în sport, Editura Universității "Alexandru Ioan Cuza" Iasi, 2005

[4] Czaplicki, A., Silva, M.; T., Ambrósio, J., A., C., Biomechanical modelling for whole body motion using natural coordinates, Journal of Theoretical And Applied Mechanics 42, 4, pp. 927-944, Warsaw, 2004. [5] Constantinescu, A., Pavel, C., Vibraţii mecanice, MATRIXRom, București, 2009.

[6] Panaitescu-Liess, R., Modelarea biomecanică a organismului uman sub acțiunea vibrațiilor, $\mathrm{PhD}$ thesis, Technical Universty for Civil Engineering, Bucharest, 2013.

[7] Picu, A. A., Modelarea biomecanică neliniară a dinamicii corpului uman sub acțiunea vibrațiilor transmise, Teză de doctorat, Galați, 2010.

[8] Pavel, C., Legendi, A., Panaitescu-Liess, R., Optimizing the functional form of a non-linear dynamic absorber attached to a nonlinear primary oscillating structure, In Proceedings of the XI-th Symposium Accoustics and Vibration of Mechanical Structure AVMS Timisoara. 26 May 2011, pp. 47-54., Politehnica, Timisoara, România.

[9] Moşneguţu, V., Chiroiu, V., Introducere în modelarea matematică a articulaţiei genunchiului, Editura Academiei Române, Bucureşti, 2013. 